\title{
Chemostats with random inputs and wall growth
}

\author{
Tomás Caraballo, Xiaoying Han* and Peter E. Kloeden
}

\author{
Dedicated to Prof. Dr. Messoud Efendiyev on occasion of his $60^{\text {th }}$ birthday
}

\begin{abstract}
Chemostat refers to a laboratory device used for growing microorganisms in a cultured environment, and has been regarded as an idealization of nature to study competition modeling of mathematical biology. The simple form of chemostat model assumes that the availability of nutrient and its supply rate are both fixed. In addition the tendency of microorganism to adhere to surfaces is neglected by assuming the flow rate is fast enough. However, these assumptions largely limit the applicability of chemostat models to realistic competition systems. In this paper, we relax these assumptions and study the chemostat models with random nutrient supplying rate or random input nutrient concentration, with or without wall growth. This leads the models to random dynamical systems and requires the concept of random attractors developed in the theory of random dynamical systems. Our results include existence of uniformly bounded non-negative solutions, existence of random attractors and geometric details of random attractors for different value of parameters. Copyright (C) 2009 John Wiley \& Sons, Ltd.
\end{abstract}

Keywords: chemostat, random input, wall growth, random dynamical system, random attractor.

\section{Introduction}

Competition modeling is one of the most challenging aspects of mathematical biology. The simplest form of competition occurs when two or more populations compete for the same resource, e.g. a common food supply of a growth-limiting nutrient. Chemostat can be regarded as a laboratory idealization of nature to study such competitions [31]. The chemostat model plays an important role in theoretical ecology $[6,10,13,16,26,27,28,30]$, waste water treatment $[1$, 21], and the study of recombinant problems in genetically altered organism $[14,15]$. Derivation and analysis of chemostat models are well documented in $[23,24,29]$ and references therein.

Two standard assumptions for simple chemostat models are: (1) the availability of the nutrient and its supply rate are fixed and (2) the tendency of microorganism to adhere to surfaces is not taken into account. Denoting by $x(t)$ the concentration of the growth-limiting nutrient and $y(t)$ the concentration of the microorganism at any specific time $t$, these assumptions lead to the following growth equations [22, 24]:

$$
\begin{aligned}
& x^{\prime}(t)=D(I-x)-a U(x(t)) y(t), \\
& y^{\prime}(t)=-D y(t)+a U(x(t)) y(t),
\end{aligned}
$$

where $D$ is the rate at which the nutrient is supplied and also the rate at which the contents of the growth medium are removed, $I$ is the input nutrient concentration which describes the quantity of nutrient available with the system at any time, $a$ is the maximal consumption rate of the nutrient and also the maximum specific growth rate of microorganisms, and $U$ is the functional

T. Caraballo: Dpto. Ecuaciones Diferenciales y Análisis Numérico, Universidad de Sevilla, Apdo. de Correos 1160, 41080-Sevilla, Spain (Email: caraball@us.es) X. Han: Department of Mathematics and Statistics, Auburn University, Auburn, AL 36832, US (Email: xzh0003@auburn.edu)

P. Kloeden: Institut für Mathematik, Goethe Universität, D-60054 Frankfurt am Main, Germany (Email: kloeden@math.uni-frankfurt.de)

* Correspondence to: Email: xzh0003@auburn.edu. Postal Address: 221 Parker Hall, Department of Mathematics and Statistics, Auburn University, Auburn, AL 36849 USA. Tel:+1-334-208-0225. 
response of the microorganism describing how the nutrient is consumed by the species. We assume in this paper that $U$ follows the Michaelis-Menten or Holling type-II form:

$$
U(x)=\frac{x}{m+x}
$$

where $m>0$ is the half-saturation constant $[22,24]$.

However, in reality the chemostat models are neither autonomous nor deterministic; they process information provided by physical or chemical inputs with noise, which can be caused by environmental perturbations, internal variability, randomly fluctuating parameters, measurement errors, etc. This motives our study of chemostat models with randomly fluctuated input parameters, the nutrient supplying rate $D$ and the nutrient supplying concentration I. From biological point of view, for an experiment to be repeatable, one has to have a reasonable description of its random aspects. These aspects may change in time and thus the noise can be modeled as a time-dependent stochastic process with certain known properties.

Representing mathematically such a stochastic process starts with a probability space $(\Omega, \mathcal{F}, \mathbb{P})$, where $\mathcal{F}$ is the $\sigma$-algebra of measurable subsets of $\Omega$ and $\mathbb{P}$ is the probability measure. To connect the state $\omega \in \Omega$ of the random environment with its state after a time $t$ has elapsed, we define a family of time-dependent maps $\left\{\theta_{t}: \Omega \rightarrow \Omega\right\}$ that keeps track of noise. In this work, we formalize the two input parameters $D$ as $D\left(\theta_{t} \omega\right)$ and $I$ as $I\left(\theta_{t} \omega\right)$. In addition, we formalize $D\left(\theta_{t} \omega\right)$ and $I\left(\theta_{t} \omega\right)$ as bounded processes. This is a natural formalism to model the realistic stochastic fluctuations of a biological system caused by its interaction with the external world, because the parameters in dynamical systems of biological interest are inherently positive and bounded.

One other drawback of traditional chemostat model (1)-(2) is the ignorance of wall attachment of microorganisms. Most of the time microorganisms grow not only in the growth medium, but also along the walls of the container, either due to the ability of the microorganisms to stick on to the walls of the container or the flow rate is not fast enough to wash these organisms out of the system. Naturally, we can regard the consumer population $y(t)$ as an aggregate of two categories of populations, one in the growth medium, denoted by $y_{1}(t)$, and the other on the walls of the container, denoted by $y_{2}(t)$. These individuals may switch their categories at any time, i.e., the microorganisms on the walls may join those in the growth medium or the biomass in the medium may prefer walls.

Let $r_{1}$ and $r_{2}$ represent the rates at which the organisms stick on to and shear off from the walls, respectively. Assume that the nutrient is equally available to both of the categories, therefore it is assumed that both categories consume the same amount of nutrient and at the same rate. When the flow rate is low, the organisms may die naturally before being washed out and thus washout is no longer the only prime factor of death. Denote by $\nu(>0)$ the collective death rate coefficient of $y(t)$ representing all the aforementioned factors such as diseases, aging, etc. On the other hand, when the flow rate is small, the dead biomass is not sent out of the system immediately and is subject to bacterial decomposition which in turn leads to regeneration of the nutrient. Expecting not $100 \%$ recycling of the dead material but only a fraction, we let constant $b \in(0,1)$ describe the fraction of dead biomass that is recycled.

Our object is to study the evolution of concentrations of the nutrient and microorganism when the input parameters are random and wall growth is taken into account, which can be described by the following random system:

$$
\begin{aligned}
& x^{\prime}(t)=D\left(\theta_{t} \omega\right)\left(I\left(\theta_{t} \omega\right)-x(t)\right)-a \frac{x(t)}{m+x(t)}\left(y_{1}(t)+y_{2}(t)\right)+b \nu y_{1}(t) \\
& y_{1}^{\prime}(t)=-\left(\nu+D\left(\theta_{t} \omega\right)\right) y_{1}(t)+c \frac{x(t)}{m+x(t)} y_{1}(t)-r_{1} y_{1}(t)+r_{2} y_{2}(t) \\
& y_{2}^{\prime}(t)=-\nu y_{2}(t)+c \frac{x(t)}{m+x(t)} y_{2}(t)+r_{1} y_{1}(t)-r_{2} y_{2}(t)
\end{aligned}
$$

where $0<c \leq a$ is the growth rate coefficient of the consumer species. In particular we assume that the inputs are perturbed by real noise, i.e. $D\left(\theta_{t} \omega\right)$ and $I\left(\theta_{t} \omega\right)$ are continuous and essentially bounded:

$$
D\left(\theta_{t} \omega\right) \in d \cdot[1-\varepsilon, 1+\varepsilon], \quad I\left(\theta_{t} \omega\right) \in i \cdot[1-\varepsilon, 1+\varepsilon], \quad d>0, \quad i>0, \quad \varepsilon<1 .
$$

Bounded noise can be modeled in various ways. For example in [4], given a stochastic process $Z_{t}$ such as an Ornstein-Uhlenbeck process, the stochastic process

$$
\zeta\left(Z_{t}\right):=\zeta_{0}\left(1-2 \varepsilon \frac{Z_{t}}{1+Z_{t}^{2}}\right)
$$

where $\zeta_{0}$ and $\varepsilon$ are positive constants with $\varepsilon \in(0,1]$, takes values in the interval $\zeta_{0}[1-\varepsilon, 1+\varepsilon]$ and tends to peak around $\zeta_{0}(1 \pm \varepsilon)$. It is thus suitable for a noisy switching scenario. In another example, the stochastic process

$$
\eta\left(Z_{t}\right):=\eta_{0}\left(1-\frac{2 \varepsilon}{\pi} \arctan Z_{t}\right)
$$


where $\eta_{0}$ and $\varepsilon$ are positive constants with $\varepsilon \in(0,1]$ takes values in the interval $\eta_{0}[1-\varepsilon, 1+\varepsilon]$ and is centered on $\eta_{0}$. In the theory of random dynamical systems the driving noise process $Z_{t}(\omega)$ is replaced by a canonical driving system $\theta_{t} \omega$. This simplifications allows a better understanding of the path-wise approach to model noise: a system influenced by stochastic processes for each single realization $\omega$ can be interpreted as wandering along a path $\theta_{t} \omega$ in $\Omega$ and thus may provide additional statistical/geological information to the modeler.

The rest of the paper is organized as follows. In section 2 we give a brief preliminary review of classical random dynamical system (RDS) theory, in section 3 we analyze the special case of system (4)-(6) without wall growth. In section 4 we analyze the full system of (4)-(6), with wall growth. Some closing remarks will be stated in section 5.

\section{Random dynamical systems}

In this section we first present some concepts (from [2]) related to general random dynamical systems (RDSs) and random attractors that we require in the sequel. Our situation is, in fact, somewhat simpler, but to facilitate the reader's access to the literature we give more general definitions here.

Let $\left(X,\|\cdot\|_{X}\right)$ be a separable Banach space and let $(\Omega, \mathcal{F}, \mathbb{P})$ be a probability space where $\mathcal{F}$ is the $\sigma$-algebra of measurable subsets of $\Omega$ (called "events") and $\mathbb{P}$ is the probability measure. To connect the state $\omega$ in the probability space $\Omega$ at time 0 with its state after a time of $t$ elapses, we define a flow $\theta=\left\{\theta_{t}\right\}_{t \in \mathbb{R}}$ on $\Omega$ with each $\theta_{t}$ being a mapping $\theta_{t}: \Omega \rightarrow \Omega$ that satisfies

(1) $\theta_{0}=\operatorname{ld}_{\Omega}$

(2) $\theta_{s} \circ \theta_{t}=\theta_{s+t}$ for all $s, t \in \mathbb{R}$,

(3) the mapping $(t, \omega) \mapsto \theta_{t} \omega$ is measurable and

(4) the probability measure $\mathbb{P}$ is preserved by $\theta_{t}$, i.e. $\theta_{t} \mathbb{P}=\mathbb{P}$.

This set-up establishes a time-dependent family $\theta$ that tracks the noise, and $(\Omega, \mathcal{F}, \mathbb{P}, \theta)$ is called a metric dynamical system [2]

Definition 1 A stochastic process $\{S(t, \omega)\}_{t \geq 0, \omega \in \Omega}$ is said to be a continuous $\operatorname{RDS}$ over $\left(\Omega, \mathcal{F}, \mathbb{P},\left(\theta_{t}\right)_{t \in \mathbb{R}}\right)$ with state space $X$ if $S:[0,+\infty) \times \Omega \times X \rightarrow X$ is $(\mathcal{B}[0,+\infty) \times \mathcal{F} \times \mathcal{B}(X), \mathcal{B}(X))$ - measurable, and for each $\omega \in \Omega$,

(i) the mapping $S(t, \omega): X \rightarrow X, x \mapsto S(t, \omega) x$ is continuous for every $t \geq 0$;

(ii) $S(0, \omega)$ is the identity operator on $X$;

(iii) (cocycle property) $S(t+s, \omega)=S\left(t, \theta_{s} \omega\right) S(s, \omega)$ for all $s, t \geq 0$.

Definition 2 (i) A set-valued mapping $B: \omega \rightarrow 2^{x} \backslash \emptyset$ is said to be a random set if the mapping $\omega \mapsto \operatorname{dist}_{x}(x, B(\omega))$ is measurable for any $x \in X$.

(ii) A random set $B(\omega)$ is said to be bounded if $B(\omega)$ is bounded for a.e. $\omega \in \Omega$; a random set $B(\omega)$ is said to be compact if $B(\omega)$ is compact for a.e. $\omega \in \Omega$; a random set is said to be closed if $B(\omega)$ is closed for a.e. $\omega \in \Omega$.

(iv) A bounded random set $B(\omega) \subset X$ is said to be tempered with respect to $\left(\theta_{t}\right)_{t \in \mathbb{R}}$ if for a.e. $\omega \in \Omega$,

$$
\lim _{t \rightarrow \infty} e^{-\beta t} \sup _{x \in B\left(\theta_{-t} \omega\right)}\|x\|_{x}=0, \quad \text { for all } \beta>0
$$

a random variable $\omega \mapsto r(\omega) \in \mathbb{R}$ is said to be tempered with respect to $\left(\theta_{t}\right)_{t \in \mathbb{R}}$ if for a.e. $\omega \in \Omega$,

$$
\lim _{t \rightarrow \infty} e^{-\beta t} \sup _{t \in \mathbb{R}}\left|r\left(\theta_{-t} \omega\right)\right|=0, \quad \text { for all } \beta>0 .
$$

In what follows we use $\mathcal{D}(X)$ to denote the set of all tempered random sets of $X$.

Definition $3 A$ random set $K(\omega) \subset X$ is called a random absorbing set in $\mathcal{D}(X)$ if for any $B \in \mathcal{D}(X)$ and a.e. $\omega \in \Omega$, there exists $T_{B}(\omega)>0$ such that

$$
S\left(t, \theta_{-t} \omega\right) B\left(\theta_{-t} \omega\right) \subset K(\omega), \quad \forall t \geq T_{B}(\omega)
$$

Definition 4 Let $\{S(t, \omega)\}_{t \geq 0, \omega \in \Omega}$ be an $\operatorname{RDS}$ over $\left(\Omega, \mathcal{F}, \mathbb{P},\left(\theta_{t}\right)_{t \in \mathbb{R}}\right)$ with state space $X$ and let $\mathcal{A}(\omega)(\subset X)$ be a random set. Then $\mathcal{A}(\omega)$ is called a global random $\mathcal{D}$ attractor (or pullback $\mathcal{D}$ attractor) for $\{S(t, \omega)\}_{t \geq 0, \omega \in \Omega}$ if $\omega \mapsto \mathcal{A}(\omega)$ satisfies

(i) (random compactness) $\mathcal{A}(\omega)$ is a compact set of $X$ for a.e. $\omega \in \Omega$;

(ii) (invariance) for a.e. $\omega \in \Omega$ and all $t \geq 0$, it holds

$$
S(t, \omega) \mathcal{A}(\omega)=\mathcal{A}\left(\theta_{t} \omega\right)
$$


(iii) (attracting property) for any $B \in \mathcal{D}(X)$ and a.e. $\omega \in \Omega$,

$$
\lim _{t \rightarrow \infty} \operatorname{dist}_{x}\left(S\left(t, \theta_{-t} \omega\right) B\left(\theta_{-t} \omega\right), A(\omega)\right)=0,
$$

where

$$
\operatorname{dist}_{X}(G, H)=\sup _{g \in G} \inf _{h \in H}\|g-h\|_{X}
$$

is the Hausdorff semi-metric for $G, H \subseteq X$.

Proposition $1[5,8,12]$ Let $B \in \mathcal{D}(X)$ be an absorbing set for the continuous random dynamical system $\{S(t, \omega)\}_{t \geq 0, \omega \in \Omega}$ which is closed and satisfies the asymptotic compactness condition for a.e. $\omega \in \Omega$, i.e., each sequence $x_{n} \in S\left(t_{n}, \theta_{-t_{n}}, B\left(\theta_{-t_{n}} \omega\right)\right)$ has a convergent subsequence in $X$ when $t_{n} \rightarrow \infty$. Then the cocycle $S$ has a unique global random attractor with component subsets

$$
\mathcal{A}(\omega)=\bigcap_{\tau \geq t_{B}(\omega)} \overline{\bigcup_{t \geq \tau} S\left(t, \theta_{-t} \omega\right) B\left(\theta_{-t} \omega\right)}
$$

If the pullback absorbing set is positively invariant, i.e., $S(t, \omega) B(\omega) \subset B\left(\theta_{t} \omega\right)$ ) for all $t \geq 0$, then

$$
\mathcal{A}(\omega)=\bigcap_{t \geq 0} S\left(t, \theta_{-t} \omega\right) B\left(\theta_{-t} \omega\right) .
$$

For state space $X=\mathbb{R}^{d}$ as in this paper, the asymptotic compactness follows trivially. Note that the random attractor is pathwise attracting in the pullback sense, but need not be pathwise attracting in the forward sense, although it is forward attracting in probability, due to some possible large deviations, see e.g., Arnold [2].

When the cocycle mapping is strictly uniformly contracting $[7,18]$, i.e., there exists $K>0$ such that

$$
\left\|S(t, \omega) x_{0}-S(t, \omega) y_{0}\right\|_{x} \leq e^{-K t}\left\|x_{0}-y_{0}\right\|_{x}
$$

for all $t \geq 0, \omega \in \Omega$ and $x_{0}, y_{0} \in X$, then the random attractor consists of singleton subsets $\mathcal{A}(\omega)=\{A(\omega)\}$. It is thus essentially a single stochastic process with sample paths $A\left(\theta_{t} \omega\right)$ for all $t \in \mathbb{R}$. The proof uses a Cauchy sequence rather than compactness argument. In this case the random attractor is pathwise attracting in both the pullback and forward senses.

\subsection{Chemostat generates an RDS}

We next prove that equations (4)-(6) generates a random dynamical system, and the random dynamical system has a random attractor. Letting

$$
\mathbb{R}_{+}^{3}=\left\{(x, y, z) \in \mathbb{R}^{3}: x \geq 0, y \geq 0, z \geq 0\right\}
$$

we have the following lemma.

Lemma 1 For any $\omega \in \Omega$, any $t_{0} \in \mathbb{R}$, and any initial data $u_{0}:=\left(x\left(t_{0}\right), y_{1}\left(t_{0}\right), y_{2}\left(t_{0}\right)\right)^{T} \in \mathbb{R}_{+}^{3}$, system (4)-(6) admits a unique solution $u\left(\cdot ; t_{0}, \omega, u_{0}\right) \in C\left(\left[t_{0}, \infty\right), \mathbb{R}_{+}^{3}\right)$ with $u\left(t_{0} ; t_{0}, \omega, u_{0}\right)=u_{0}$ and generates a random dynamical system $S(t, \omega)(\cdot)$ defined as

$$
S(t, \omega) u_{0}=u\left(t ; 0, \omega, u_{0}\right), \quad \forall t \geq 0, u_{0} \in \mathbb{R}_{+}^{3}, \omega \in \Omega .
$$

Moreover, $u(\cdot)=u\left(\cdot ; t_{0}, \omega, u_{0}\right)$ is bounded.

Proof. With $u(t)=\left(x(t), y_{1}(t), y_{2}(t)\right)^{T}$, system (4)-(6) can be written as

$$
\frac{d u}{d t}=L\left(\theta_{t} \omega\right) \cdot u+F\left(u, \theta_{t} \omega\right),
$$

where

$$
L\left(\theta_{t} \omega\right)=\left(\begin{array}{ccc}
-D\left(\theta_{t} \omega\right) & 0 & 0 \\
0 & -\nu-D\left(\theta_{t} \omega\right)-r_{1} & r_{2} \\
0 & r_{1} & -\nu-r_{2}
\end{array}\right),
$$

and $F: \mathbb{R}_{+}^{3} \times\left[t_{0},+\infty\left(\rightarrow \mathbb{R}^{3}\right.\right.$ is given by

$$
F\left(v, \theta_{t} \omega\right)=\left(\begin{array}{c}
D\left(\theta_{t} \omega\right) I\left(\theta_{t} \omega\right)-\frac{a v_{1}}{m+v_{1}}\left(v_{2}+v_{3}\right) \\
\frac{c v_{1}}{m+v_{1}} v_{2} \\
\frac{c v_{1}}{m+v_{1}} v_{3}
\end{array}\right),
$$


where $v=\left(v_{1}, v_{2}, v_{3}\right) \in \mathbb{R}_{+}^{3}$

Since $D\left(\theta_{t} \omega\right)$ is bounded, the operator $L$ generates an evolution system on $\mathbb{R}^{3}$. Also because $D\left(\theta_{t} \omega\right)$ and $I\left(\theta_{t} \omega\right)$ are continuous with respect to $t$, function $F(\cdot, \theta . \omega) \in C\left(\mathbb{R}_{+}^{3} \times\left[t_{0}, \infty\right), \mathbb{R}^{3}\right)$ and is continuously differentiable with respect to the variables $\left(v_{1}, v_{2}, v_{3}\right)$, being the partial derivatives bounded inside $\mathbb{R}_{+}^{3}$, what implies that is globally Lipschitz with respect to $\left(v_{1}, v_{2}, v_{3}\right)$ in $\mathbb{R}_{+}^{3}$. Therefore, thanks to the classical results from the theory of ordinary differential equations, system (4)-(6) possesses a unique global solution $u\left(\cdot ; t_{0}, \omega, u_{0}\right) \in C^{1}\left(\left[t_{0}, \infty\right), \mathbb{R}^{3}\right)$

Notice that it is straightforward to check that

$$
u\left(t+t_{0} ; t_{0}, \omega, u_{0}\right)=u\left(t ; 0, \theta_{t_{0}} \omega, u_{0}\right)
$$

for all $t_{0} \in \mathbb{R}, t \geq t_{0}, \omega \in \Omega, u_{0} \in \mathbb{R}_{+}^{3}$. This allows us to define a mapping $S(t, \omega)(\cdot)$, which will be our random dynamical system, as

$$
S(t, \omega) u_{0}=u\left(t ; 0, \omega, u_{0}\right), \quad \forall t \geq 0, u_{0} \in \mathbb{R}_{+}^{3}, \omega \in \Omega .
$$

From now on, we will simply write $u\left(t ; \omega, u_{0}\right)$ instead of $u\left(t ; 0, \omega, u_{0}\right)$.

Now, as we will argue for each $\omega \in \Omega$ fixed, we will not mention explicitly the random parameter and will write $u(t)=$ $u\left(t ; \omega, u_{0}\right)$, and the same as far as its components are involved. By continuity of solutions, each solution has to take value 0 before it reaches a negative value. With $x=0$ and $y_{1}, y_{2} \geq 0$, equation (4) becomes

$$
x^{\prime}(t)=D\left(\theta_{t} \omega\right) /\left(\theta_{t} \omega\right)+b \nu y_{1}(t)>0
$$

and thus $x(t)$ is strictly increasing at $x=0$. Similarly, with $y_{1}=0$ and $x, y_{2} \geq 0$, equation (5) gives $y_{1}^{\prime}(t)=r_{2} y_{2} \geq 0$, and with $y_{2}=0$ and $x, y_{1} \geq 0$, equation (6) gives $y_{2}^{\prime}(t)=r_{1} y_{1} \geq 0$. Therefore, $y_{1}(t)$ is non-decreasing at $y_{1}=0$ and $y_{2}(t)$ is non-decreasing at $y_{2}=0$, i.e., $u(t) \in \mathbb{R}_{+}^{3}$ for any $t \geq 0$.

For any $u_{0} \in \mathbb{R}_{+}^{3}$, solution $u\left(\cdot ; \omega, u_{0}\right) \in \mathbb{R}_{+}^{3}$ for $t \in[0, \infty)$. Since function $F\left(u, \theta_{t} \omega\right)=F(u, t, \omega)$ is continuous in $u$, $t$, and is measurable in $\omega, u:[0, \infty) \times \Omega \times \mathbb{R}_{+}^{3} \rightarrow \mathbb{R}_{+}^{3},\left(t ; \omega, u_{0}\right) \mapsto u\left(t ; \omega, u_{0}\right)$ is $\left(\mathcal{B}[0, \infty) \times \mathcal{F} 0 \times \mathcal{B}\left(\mathbb{R}_{+}^{3}\right), \mathcal{B}\left(\mathbb{R}_{+}^{3}\right)\right)$-measurable. It then follows directly that (4)-(6) generate the continuous random dynamical system $S(t, \omega)(\cdot)$ defined by $(9)$.

For $u(t) \in \mathbb{R}_{+}^{3}$, define $\|u(t)\|_{1}:=x(t)+y_{1}(t)+y_{2}(t)$, and let $s(t)=x(t)+\frac{a}{c}\left(y_{1}(t)+y_{2}(t)\right)$. Since $a \geq c$, then

$$
\|u(t)\|_{1} \leq s(t) \leq \frac{a}{c} \cdot\|u(t)\|_{1}
$$

The time derivative of $s(t)$ along solutions to (4)-(6) satisfies

$$
\begin{aligned}
\frac{d s(t)}{d t} & =D\left(\theta_{t} \omega\right)\left[I\left(\theta_{t} \omega\right)-x(t)\right]-\left[\frac{a}{c}\left(\nu+D\left(\theta_{t} \omega\right)\right)-b \nu\right] y_{1}(t)-\frac{a}{c} \nu y_{2}(t) \\
& \leq d i(1+\varepsilon)^{2}-d(1-\varepsilon) x(t)-\left[\frac{a}{c}(\nu+d(1-\varepsilon))-b \nu\right] y_{1}(t)-\frac{a}{c} \nu y_{2}(t) .
\end{aligned}
$$

Note that, since $a \geq c$ and $0<b<1$,

$$
\frac{a}{c}(\nu+d(1-\varepsilon)-b \nu)=\frac{a}{c} d(1-\varepsilon)+\left(\frac{a}{c}-b\right) \nu>\frac{a}{c} d(1-\varepsilon) .
$$

Therefore by letting $\lambda:=\min \{d(1-\varepsilon), \nu\}$ we obtain

$$
\frac{d s(t)}{d t} \leq d i(1+\varepsilon)^{2}-\lambda s(t)
$$

On the one hand, for $s(0) \geq d i(1+\varepsilon)^{2} / \lambda, s(t)$ will be non-increasing for all $t \geq 0$ and thus $s(t) \leq v(0)$. On the other hand, for $s(0)<d i(1+\varepsilon)^{2} / \lambda, s(t) \leq d i(1+\varepsilon)^{2} / \lambda$ for all $t \geq 0$. These imply that $\|u(t)\|_{1}$ is bounded:

$$
0 \leq\|u(t)\|_{1} \leq \max \left\{\operatorname{di}(1+\varepsilon)^{2} / \lambda, x(0)+\frac{a}{c}\left(y_{1}(0)+y_{2}(0)\right)\right\}, \quad t \geq 0 .
$$

\subsection{Existence of a random attractor}

In this subsection we study the existence of tempered random bounded absorbing sets of the random dynamical system $\{S(t, \omega)\}_{t \geq 0, \omega \in \Omega}$, followed by the existence of a random attractor for $\{S(t, \omega)\}_{t \geq 0, \omega \in \Omega \text {. }}$

Lemma 2 For $\omega \in \Omega$, there exists a tempered bounded closed random absorbing set $K(\omega) \in \mathcal{D}\left(\mathbb{R}_{+}^{3}\right)$ of the random dynamical system $\{S(t, \omega)\}_{t \geq 0, \omega \in \Omega}$ such that for any $B \in \mathcal{D}\left(\mathbb{R}_{+}^{3}\right)$ and each $\omega \in \Omega$, there exists $T_{B}(\omega)>0$ yielding

$$
S\left(t, \theta_{-t} \omega\right) B\left(\theta_{-t} \omega\right) \subset K(\omega) \quad \forall t \geq T_{B}(\omega) .
$$


Proof. Recall that $u\left(t ; \omega, u_{0}\right)=S(t, \omega) u_{0}$ denotes the solution of system (4)-(6) satisfying $u\left(0 ; \omega, u_{0}\right)=u_{0}$. Then, for $u_{0}:=u_{0}\left(\theta_{-t} \omega\right) \in B\left(\theta_{-t} \omega\right)$,

$$
\left\|S\left(t, \theta_{-t} \omega\right) u_{0}\right\|_{1}=\left\|u\left(t ; \theta_{-t} \omega, u_{0}\left(\theta_{-t} \omega\right)\right)\right\|_{1} \leq s\left(t ; \theta_{-t} \omega, s_{0}\left(\theta_{-t} \omega\right)\right) .
$$

According to inequality (11),

$$
s(t, \omega) \leq s_{0} e^{-\lambda t}+d i(1+\varepsilon)^{2} / \lambda .
$$

Substituting $\omega$ by $\theta_{-t} \omega$ in (12), we obtain

$$
s\left(t ; \theta_{-t} \omega, s_{0}\left(\theta_{-t} \omega\right)\right) \leq e^{-\lambda t} \sup _{\left(x, y_{1}, y_{2}\right) \in B\left(\theta_{-t} \omega\right)}\left(x+\frac{a}{c}\left(y_{1}+y_{2}\right)\right)+\frac{d i(1+\varepsilon)^{2}}{\lambda} .
$$

Therefore for any $\epsilon>0$, there exists $T_{B}(\omega)$ such that when $t>T_{B}$,

$$
\begin{aligned}
\left\|u\left(t ; \theta_{-t} \omega, u_{0}\right)\right\|_{1} & =x\left(t ; \theta_{-t} \omega, u_{0}\right)+y_{1}\left(t ; \theta_{-t} \omega, u_{0}\right)+y_{2}\left(t ; \theta_{-t} \omega, u_{0}\right) \\
\leq & d i(1+\varepsilon)^{2} / \lambda+\epsilon,
\end{aligned}
$$

for all $u_{0} \in B\left(\theta_{-t} \omega\right)$.

Name

$$
K_{\epsilon}(\omega)=\overline{\left\{\left(x, y_{1}, y_{2}\right) \in \mathbb{R}_{+}^{3}: x+y_{1}+y_{2} \leq d i(1+\varepsilon)^{2} / \lambda+\epsilon\right\}},
$$

then $K_{\epsilon}(\omega)$ is positively invariant and absorbing in $\mathbb{R}_{+}^{3}$.

It follows directly from Proposition 1, that the random dynamical system generated by system (4)-(6) possesses a random attractor $\mathcal{A}=\{A(\omega): \omega \in \Omega\}$, consisting of nonempty compact random subsets of $\mathbb{R}_{+}^{3}$ contained in $K_{\epsilon}(\omega)$. In the next sections, we will study the geometric structure of the random attractor of the random dynamical system generated by (4)-(6), first without wall growth, then with wall growth.

\section{Random chemostat without wall growth}

In this section we first consider the special case when $y_{2}(t) \equiv 0, r_{1}=r_{2}=0, \nu=0$ and $a=c$, which describes the random chemostat system with no wall growth. In particular we will study the system with both random nutrient input concentration and random nutrient supplying rate.

When there is no wall growth, equations (4) - (6) can be reduced to

$$
\begin{aligned}
\frac{d x(t)}{d t} & =D\left(\theta_{t} \omega\right)\left(I\left(\theta_{t} \omega\right)-x(t)\right)-a \frac{x(t)}{m+x(t)} y_{1}(t) \\
\frac{d y_{1}(t)}{d t} & =-D\left(\theta_{t} \omega\right) y_{1}(t)+a \frac{x(t)}{m+x(t)} y_{1}(t)
\end{aligned}
$$

Let $v(t):=x(t)+y_{1}(t)$, then $v(t)$ satisfies

$$
\frac{d v(t)}{d t}=D\left(\theta_{t} \omega\right)\left[I\left(\theta_{t} \omega\right)-v(t)\right]
$$

This has a nontrivial random solution which is both forward and pullback attracting. In fact, for any initial value $v_{0}$, it holds

$$
v\left(t ; \omega, v_{0}\right)=v_{0} e^{-\int_{0}^{t} D\left(\theta_{s} \omega\right) d s}+\int_{0}^{t} D\left(\theta_{s} \omega\right) /\left(\theta_{s} \omega\right) e^{-\int_{s}^{t} D\left(\theta_{\tau} \omega\right) d \tau} d s .
$$

Replacing $\omega$ by $\theta_{-t} \omega$ in (17) we obtain

$$
v\left(t ; \theta_{-t} \omega, v_{0}\right)=v_{0} e^{-\int_{-t}^{0} D\left(\theta_{s} \omega\right) d s}+\int_{-t}^{0} D\left(\theta_{s} \omega\right) I\left(\theta_{s} \omega\right) e^{-\int_{0}^{t} D\left(\theta_{\tau} \omega\right) d \tau} d s,
$$

which is pullback convergent (i.e., when $t \rightarrow+\infty$ ) to

$$
v^{*}(\omega):=\int_{-\infty}^{0} D\left(\theta_{s} \omega\right) /\left(\theta_{s} \omega\right) e^{-\int_{s}^{0} D\left(\theta_{\tau} \omega\right) d \tau} d s .
$$

Note that since $d(1-\varepsilon) \leq D\left(\theta_{t} \omega\right) \leq d(1+\varepsilon)$ and $i(1-\varepsilon) \leq I\left(\theta_{t} \omega\right) \leq i(1+\varepsilon)$,

$$
d i(1-\varepsilon)^{2} \int_{-\infty}^{0} e^{-\int_{s}^{0} d(1+\varepsilon) d \tau} d s \leq v^{*}(\omega) \leq d i(1+\varepsilon)^{2} \int_{-\infty}^{0} e^{-\int_{s}^{0} d(1-\varepsilon) d \tau} d s,
$$


i.e.

$$
\frac{i(1-\varepsilon)^{2}}{1+\varepsilon} \leq v^{*}(\omega) \leq \frac{i(1+\varepsilon)^{2}}{1-\varepsilon}, \quad \omega \in \Omega .
$$

For a fixed and small enough $\delta>0$, name the nonempty compact set

$$
K(\omega):=\left\{\left(x, y_{1}\right) \in \mathbb{R}_{+}^{2}: \frac{i(1-\varepsilon)^{2}}{1+\varepsilon}-\delta \leq x+y_{1} \leq \frac{i(1+\varepsilon)^{2}}{1-\varepsilon}+\delta\right\}
$$

Then for any $B(\omega) \in \mathcal{D}\left(\mathbb{R}_{+}^{2}\right)$ and a.e. $\omega \in \Omega$, there exists $T_{B}(\omega)>0$ such that

$$
S\left(t, \theta_{-t} \omega\right) B\left(\theta_{-t} \omega\right) \subset K(\omega),
$$

i.e. $K(\omega)$ is positively invariant and absorbing in $\mathbb{R}_{+}^{2}$ for system (16).

The random dynamical system generated by system (14)-(15) thus has a random attractor $\mathcal{A}=\{A(\omega): \omega \in \Omega\}$ consisting of non-empty compact subsets of $K(\omega)$. We provide in the next theorem sufficient conditions for the extinction and persistence of microorganism $y_{1}$.

Theorem 1 The random pullback attractor $\mathcal{A}=\{A(\omega): \omega \in \Omega\}$ for the random dynamical system generated by equations (14)-(15)

(i) has singleton component sets $A(\omega)=\left\{\left(v^{*}(\omega), 0\right)\right\}$ for every $\omega \in \Omega$, provided

$$
d(1-\varepsilon) \geq a
$$

(ii) possesses nontrivial component sets which include $\left(v^{*}(\omega), 0\right)$ and strictly positive points, provided

$$
d(1+\varepsilon) \leq \frac{a i(1-\varepsilon)^{3}}{m\left(1-\varepsilon^{2}\right)+i(1+\varepsilon)^{3}} ;
$$

(iii) contains a nontrivial entire solution that attracts all other strictly positive entire solutions, provided

$$
d(1-\varepsilon)>a-\frac{\operatorname{ami}(1-\varepsilon)^{2} /(1+\varepsilon)}{\left(m+i(1+\varepsilon)^{2} /(1-\varepsilon)\right)^{2}} .
$$

Proof. Note that since the pullback attractor contains all bounded entire solutions, then $\left(v^{*}(\omega), 0\right) \in A(\omega)$ for every $\omega \in \Omega$,.

(i) Equation (15) can be written as

$$
\begin{aligned}
\frac{d y_{1}(t)}{d t} & =\left(-D\left(\theta_{t} \omega\right)+\frac{a x(t)}{m+x(t)}\right) y_{1}(t) \\
& <(-d(1-\varepsilon)+a) y_{1}(t) .
\end{aligned}
$$

Thus when $d(1-\varepsilon)>a, y^{\prime}(t)<0$. This implies that $y_{1}(t) \rightarrow 0$ as $t \rightarrow \infty$ and $x(t, \omega)=v^{*}\left(\theta_{t} \omega\right)$, $y_{1}(t)=0$ is asymptotically stable in $\mathbb{R}_{+}^{2}$.

(ii) Besides $x+y_{1}=i(1+\varepsilon)^{2} /(1-\varepsilon)$ and $x+y_{1}=i(1-\varepsilon)^{2} /(1+\varepsilon)$, the absorbing set $K(\omega)$ has two other edges $x=0$ and $y_{1}=0$. On $x=0,\left.x^{\prime}(t)\right|_{x=0}=D\left(\theta_{t} \omega\right) /\left(\theta_{t} \omega\right)>0$, i.e., $x(t)$ is increasing towards the interior of $K(\omega)$ on this edge. The edge $y_{1}=0$ is invariant, as $\left.y_{1}^{\prime}\right|_{y_{1}=0}=0$. But for $y=\epsilon \ll i(1-\varepsilon)^{2} /(1+\varepsilon), x(t)$ satisfies $i(1-\varepsilon)^{2} /(1+\varepsilon) \leq x \leq$ $i(1+\varepsilon)^{2} /(1-\varepsilon)$. Hence when

$$
d(1+\varepsilon) \leq \frac{a i(1-\varepsilon)^{3}}{m\left(1-\varepsilon^{2}\right)+i(1+\varepsilon)^{3}}
$$

equation (15) gives

$$
\frac{d y_{1}}{d t}=\left(-D\left(\theta_{t} \omega\right)+\frac{a x(t)}{m+x(t)}\right) y_{1}>\left(\frac{a i(1-\varepsilon)^{3}}{m\left(1-\varepsilon^{2}\right)+i(1+\varepsilon)^{3}}-d(1+\varepsilon)\right) y_{1} \geq 0
$$

This implies that the positive interior of the absorbing set also contains points of the random attractor.

(iii) We now consider equation (14) restricted to the stable manifold $x(t)+y_{1}(t)=v^{*}\left(\theta_{t} \omega\right)$, on which

$$
\frac{d x(t)}{d t}=D\left(\theta_{t} \omega\right)\left(I\left(\theta_{t} \omega\right)-x(t)\right)-\frac{a x(t)}{m+x(t)}\left(v^{*}\left(\theta_{t} \omega\right)-x(t)\right) .
$$

For any two solutions $x_{1}(t)$ and $x_{2}(t)$ to equation (19), define $\delta(t)=x_{1}(t)-x_{2}(t)$. Then $\delta(t)$ satisfies

$$
\begin{aligned}
\frac{d \delta(t)}{d t} & =-D\left(\theta_{t} \omega\right) \delta(t)-a v^{*}\left(\theta_{t} \omega\right)\left(\frac{x_{1}}{m+x_{1}}-\frac{x_{2}}{m+x_{2}}\right)+a\left(\frac{x_{1}^{2}}{m+x_{1}}-\frac{x_{2}^{2}}{m+x_{2}}\right) \\
& =-D\left(\theta_{t} \omega\right) \delta(t)-\frac{a m v^{*}\left(\theta_{t} \omega\right)}{\left(m+x_{1}\right)\left(m+x_{2}\right)} \delta(t)+a \frac{m\left(x_{1}+x_{2}\right)+x_{1} x_{2}}{\left(m+x_{1}\right)\left(m+x_{2}\right)} \delta(t)
\end{aligned}
$$


Since for $t$ large enough, $x_{1}(t), x_{2}(t) \leq v^{*}\left(\theta_{t} \omega\right) \leq i(1+\varepsilon)^{2} /(1-\varepsilon)$, and $v^{*}\left(\theta_{t} \omega\right) \geq i(1-\varepsilon)^{2} /(1+\varepsilon)$, we have

$$
\frac{d \delta(t)}{d t}<-d(1-\varepsilon) \delta(t)-\frac{a m i(1-\varepsilon)^{2} /(1+\varepsilon)}{\left(m+i(1+\varepsilon)^{2} /(1-\varepsilon)\right)^{2}} \delta(t)+a \delta(t) .
$$

Hence $\delta(t) \rightarrow 0$ as $t \rightarrow \infty$ when

$$
d(1-\varepsilon)+\frac{a m i(1-\varepsilon)^{2} /(1+\varepsilon)}{\left(m+i(1+\varepsilon)^{2} /(1-\varepsilon)\right)^{2}}>a
$$

This always holds if $d(1-\varepsilon) \geq a$, which is scenario (i) of the theorem. But it can still hold if $a$ is slightly larger. In fact,

$$
\frac{m i(1-\varepsilon)^{2} /(1+\varepsilon)}{\left(m+i(1+\varepsilon)^{2} /(1-\varepsilon)\right)^{2}}<\frac{m i}{(m+i)^{2}}<1
$$

In this case the above estimates with neither $x_{1}(t)$ or $x_{2}(t)$ equals $v^{*}\left(\theta_{t} \omega\right)$, the system is strict uniformly contracting $[7,17]$ in the positive quadrant and thus has a unique entire solution $v^{*}\left(\theta_{t} \omega\right)$ as its pullback attractor in the positive quadrant.

\section{Random chemostat with wall growth}

In this section we consider the full random chemostat system with wall growth taken into account. In particular we will study the random dynamical system generated by (4)-(6) with both random nutrient input concentration and random nutrient supplying rate. To obtain more detailed information on the internal structure of the pullback attractor, we make the following change of variables:

$$
y(t)=y_{1}(t)+y_{2}(t) ; \quad \gamma(t)=\frac{y_{1}(t)}{y(t)} .
$$

System (4)-(6) then becomes

$$
\begin{aligned}
\frac{d x(t)}{d t} & =D\left(\theta_{t} \omega\right)\left(I\left(\theta_{t} \omega\right)-x(t)\right)-\frac{a x(t)}{m+x(t)} y(t)+b \nu \gamma(t) y(t), \\
\frac{d y(t)}{d t} & =-\nu y(t)-D\left(\theta_{t} \omega\right) \gamma(t) y(t)+\frac{c x(t)}{m+x(t)} y(t), \\
\frac{d \gamma(t)}{d t} & =-D\left(\theta_{t} \omega\right) \gamma(t)(1-\gamma(t))-r_{1} \gamma(t)+r_{2}(1-\gamma(t)) .
\end{aligned}
$$

By definition, $\gamma(t)$ represents the portion of microorganism that attaches to the wall. Noting that the dynamics of $\gamma(t)=$ $\gamma\left(t ; \omega, \gamma_{0}\right)$ are uncoupled with $x(t)$ and $y(t)$, we first study the dynamics of $\gamma(t)$.

\subsection{Global dynamics of $\gamma(t)$}

For any $y_{1}>0$ and $y_{2}>0$, we have $0<\gamma(t)<1$ for all $t \in \mathbb{R}$. Moreover, since

$$
\left.\frac{d \gamma}{d t}\right|_{\gamma=0}=r_{2}>0 ;\left.\quad \frac{d \gamma}{d t}\right|_{\gamma=1}=-r_{1}<0,
$$

the interval $(0,1)$ is positively invariant for $\gamma$. Therefore equation (23) has a pullback attractor $\mathcal{A}_{\gamma}=\left\{A_{\gamma}(\omega)\right\}_{\omega \in \Omega}[19]$ with its component subsets given by

$$
A_{\gamma}(\omega)=\bigcap_{t \geq 0} \gamma\left(t ; \theta_{-t} \omega,[0,1]\right)
$$

These component subsets have the form

$$
A_{\gamma}\left(\theta_{t} \omega\right)=\left[\gamma_{l}\left(\theta_{t} \omega\right), \gamma_{u}\left(\theta_{t} \omega\right)\right]
$$

where $\gamma_{l}\left(\theta_{t} \omega\right)$ and $\gamma_{u}\left(\theta_{t} \omega\right)$ are entire bounded solutions of equation (23). The other bounded entire solutions of (23) lie between these two. We next estimate bounds of these entire solutions by using differential inequalities.

On the one hand, since $\gamma(t) \leq 1$ and $D\left(\theta_{t} \omega\right)>0$, we have

$$
\begin{aligned}
\gamma^{\prime}(t) & =D\left(\theta_{t} \omega\right)\left(\gamma^{2}(t)-\gamma(t)\right)-\left(r_{1}+r_{2}\right) \gamma(t)+r_{2} \\
& \leq-\left(r_{1}+r_{2}\right) \gamma(t)+r_{2} .
\end{aligned}
$$

On the other hand,

$$
\begin{aligned}
\gamma^{\prime}(t) & =D\left(\theta_{t} \omega\right) \gamma^{2}(t)-\left(D\left(\theta_{t} \omega\right)+r_{1}+r_{2}\right) \gamma(t)+r_{2} \\
& \geq-\left(d(1+\varepsilon)+r_{1}+r_{2}\right) \gamma(t)+r_{2}
\end{aligned}
$$


Let $\alpha(t)$ and $\beta(t)$ satisfy

$$
\begin{aligned}
& \alpha(t)=-\left(d(1+\varepsilon)+r_{1}+r_{2}\right) \alpha(t)+r_{2}, \quad \alpha(0)=\gamma(0), \\
& \beta(t)=-\left(r_{1}+r_{2}\right) \beta(t)+r_{2}, \quad \beta(0)=\gamma(0) .
\end{aligned}
$$

Then $\alpha(t) \leq \gamma(t) \leq \beta(t)$ and $\left[\gamma_{l}\left(\theta_{t} \omega\right), \gamma_{u}\left(\theta_{t} \omega\right)\right] \subseteq\left[\alpha^{*}, \beta^{*}\right]$, where

$$
\alpha^{*}=\frac{r_{2}}{r_{1}+r_{2}+d(1+\varepsilon)}, \quad \beta^{*}=\frac{r_{2}}{r_{1}+r_{2}}
$$

are asymptotically stable steady states for (24) and (25) respectively. In summary,

$$
A_{\gamma}(\omega)=\left[\gamma_{l}(\omega), \gamma_{u}(\omega)\right] \subseteq\left[\alpha^{*}, \beta^{*}\right]
$$

We provide the sufficient condition for $\mathcal{A}$ to consist of only a single entire solution in the next theorem.

Theorem 2 The pullback attractor $\mathcal{A}$ associated to the random dynamical system $\gamma(t, \omega, \cdot)$ generated by (23) consists of a single entire solution, denoted by $\gamma^{*}\left(\theta_{t} \omega\right)$, provided

$$
2 r_{2} d(1+\varepsilon)<\left(r_{1}+r_{2}+d(1-\varepsilon)\right)\left(r_{1}+r_{2}\right)
$$

Proof. Equivalently, we need to find conditions under which $\gamma_{l}\left(\theta_{t} \omega\right) \equiv \gamma_{u}\left(\theta_{t} \omega\right), t \geq 0$. To this end, we consider the difference between $\gamma_{l}\left(\theta_{t} \omega\right)$ and $\gamma_{u}\left(\theta_{t} \omega\right), h(t):=\gamma_{u}\left(\theta_{t} \omega\right)-\gamma_{l}\left(\theta_{t} \omega\right)$. Then $h$ satisfies

$$
\frac{d h(t)}{d t}=D\left(\theta_{t} \omega\right)\left(\gamma_{l}\left(\theta_{t} \omega\right)+\gamma_{u}\left(\theta_{t} \omega\right)\right) h(t)-\left(D\left(\theta_{t} \omega\right)+r_{1}+r_{2}\right) h(t)
$$

Solving (27) and replacing $\omega$ by $\theta_{-t} \omega$ we obtain

$$
\begin{aligned}
h\left(t, \theta_{-t} \omega\right) & =h_{0} e^{\int_{-t}^{0} D\left(\theta_{s} \omega\right)\left(\gamma_{l}\left(\theta_{s} \omega\right)+\gamma_{u}\left(\theta_{s} \omega\right)\right)-\left(D\left(\theta_{s} \omega\right)+r_{1}+r_{2}\right) d s} \\
& \leq h_{0} e^{\int_{-t}^{0} d(1+\varepsilon) \cdot 2 \gamma_{u}\left(\theta_{s} \omega\right)-\left(d(1-\varepsilon)+r_{1}+r_{2}\right)} \\
& \leq e^{\left(\frac{2 d r_{2}(1+\varepsilon)}{r_{1}+r_{2}}-\left(r_{1}+r_{2}+d(1-\varepsilon)\right)\right) t} .
\end{aligned}
$$

Therefore when $2 r_{2} d(1+\varepsilon)<\left(r_{1}+r_{2}+d(1-\varepsilon)\right)\left(r_{1}+r_{2}\right), h\left(t, \theta_{-t} \omega\right) \rightarrow 0$ as $t \rightarrow \infty$. This implies that the pullback attractor $\mathcal{A}$ for the random dynamical system $\gamma(t, \omega, \cdot)$ has only one single entire solution.

Note that this sufficient condition is equivalent to

$$
d \varepsilon<\frac{\left(r_{1}+r_{2}\right)^{2}+d\left(r_{1}-r_{2}\right)}{r_{1}+3 r_{2}}
$$

which essentially represents the restriction on the magnitude of noise on $D$. We next study the dynamics of $x(t)$ and $y(t)$.

\subsection{Global dynamics of $x(t)$ and $y(t)$}

For convenience for readers, we restate equations for $x(t)$ and $y(t)$ here:

$$
\begin{aligned}
\frac{d x(t)}{d t} & =D\left(\theta_{t} \omega\right)\left(I\left(\theta_{t} \omega\right)-x(t)\right)-\frac{a x(t)}{m+x(t)} y(t)+b \nu \gamma(t) y(t), \\
\frac{d y(t)}{d t} & =-\nu y(t)-D\left(\theta_{t} \omega\right) \gamma(t) y(t)+\frac{c x(t)}{m+x(t)} y(t) .
\end{aligned}
$$

According to the dynamics of $\gamma(t)$ discussed in the previous subsection, for $t$ large enough, $\gamma(t)$ satisfies

$$
\alpha^{*} \leq \gamma(t) \leq \beta^{*}
$$

where $\alpha^{*}$ and $\beta^{*}$ are as stated in (26).

Theorem 3 Given $a \geq c, 0<b<1, \nu>0$, assume that $D\left(\theta_{t} \omega\right)$ and $I\left(\theta_{t} \omega\right)$ are continuous and essentially bounded, with $d(1-\varepsilon) \leq D\left(\theta_{t} \omega\right) \leq d(1+\varepsilon)$ and $i(1-\varepsilon) \leq I\left(\theta_{t} \omega\right) \leq i(1+\varepsilon)$. Then, system (21)-(22) has a pullback attractor $\mathcal{A}=\{A(\omega)$ : $\omega \in \Omega\}$ inside the nonnegative quadrant. Moreover, letting

$$
x^{*}(\omega)=\int_{-\infty}^{0} D\left(\theta_{s} \omega\right) /\left(\theta_{s} \omega\right) e^{-\int_{s}^{0} D\left(\theta_{\tau} \omega\right) d \tau} d s
$$


(i) the pullback attractor $\mathcal{A}$ has a singleton component subset $A(\omega)=\left\{\left(x^{*}(\omega), 0\right)\right\}$ provided

$$
\nu+d(1-\varepsilon) \alpha^{*} \geq c
$$

(ii) the pullback attractor $\mathcal{A}$ also contains points strictly inside the positive quadrant in addition to the singleton solution $\left\{\left(x^{*}(\omega), 0\right)\right\}$ provided

$$
a c^{2} d i(1-\varepsilon)^{2}>\left(m\left(a \nu+a d(1+\varepsilon)-c b \nu \beta^{*}\right)+\operatorname{acdi}(1-\varepsilon)^{2}\right) \cdot\left(\nu+d(1+\varepsilon) \beta^{*}\right) .
$$

Proof. Let $z(t):=c x(t)+a y(t)$, then $z(t)$ satisfies

$$
\frac{d z(t)}{d t}=c D\left(\theta_{t} \omega\right) I\left(\theta_{t} \omega\right)-D\left(\theta_{t} \omega\right) \cdot c x(t)-\left[\nu+D\left(\theta_{t} \omega\right) \gamma(t)-\frac{c b \nu}{a} \gamma(t)\right] \cdot a y(t)
$$

Note that since $b<1, c \leq a$ and $\gamma(t) \leq \beta^{*}<1$, then

$$
\nu+D\left(\theta_{t} \omega\right) \gamma(t)-\frac{c b \nu}{a} \gamma(t) \geq D\left(\theta_{t} \omega\right) \gamma(t) .
$$

Therefore

$$
\begin{aligned}
\frac{d z(t)}{d t} & \leq c D\left(\theta_{t} \omega\right) /\left(\theta_{t} \omega\right)-D\left(\theta_{t} \omega\right) \gamma(t) z(t) \\
& \leq c d i(1+\varepsilon)^{2}-d(1-\varepsilon) \alpha^{*} z(t),
\end{aligned}
$$

and

$$
z(t) \leq \frac{c i(1+\varepsilon)^{2}}{(1-\varepsilon) \alpha^{*}}=\frac{c i(1+\varepsilon)^{2}\left(r_{1}+r_{2}+d(1+\varepsilon)\right)}{(1-\varepsilon) r_{2}} .
$$

It follows that for $0<\varepsilon<1$, the nonempty compact set

$$
K(\omega):=\left\{(x, y) \in \mathbb{R}_{+}^{2}: 0 \leq c x+a y \leq \frac{c i(1+\varepsilon)^{2}\left(r_{1}+r_{2}+d(1+\varepsilon)\right)}{(1-\varepsilon) r_{2}}\right\}
$$

is positively invariant and absorbing in $\mathbb{R}_{+}^{2}$. The random dynamical system on $\mathbb{R}_{+}^{2}$ generated by equations (21)-(22) thus has a pullback attractor $\mathcal{A}=\{A(\omega): \omega \in \Omega\}$, consisting of nonempty compact subsets of $\mathbb{R}_{+}^{2}$ that are contained in $K(\omega)$.

(i) When $\nu+d(1-\varepsilon) \alpha^{*} \geq c, y(t)$ satisfies

$$
\begin{aligned}
\frac{d y(t)}{d t} & =-\left(\nu+D\left(\theta_{t} \omega\right) \gamma(t)-\frac{c x(t)}{m+x(t)}\right) y(t) \\
& <-\left(\nu+d(1-\varepsilon) \alpha^{*}-c\right) y(t) \leq 0 .
\end{aligned}
$$

Thus $y(t)$ decreases to 0 as $t$ approaches $\infty$. Consequently, $x(t)$ satisfies

$$
\frac{d x(t)}{d t}=D\left(\theta_{t} \omega\right)\left(I\left(\theta_{t} \omega\right)-x(t)\right)
$$

The solution to $(32)$ is

$$
x(t)=x(0) e^{-\int_{0}^{t} D\left(\theta_{s} \omega\right) d s}+\int_{0}^{t} D\left(\theta_{s} \omega\right) /\left(\theta_{s} \omega\right) e^{-\int_{s}^{t} D\left(\theta_{\tau} \omega\right) d \tau} d s .
$$

Replacing $\omega$ by $\theta_{-t} \omega$, we obtain

$$
x\left(t, \theta_{-t} \omega\right)=x(0) e^{-\int_{-t}^{0} D\left(\theta_{s} \omega\right) d s}+\int_{-t}^{0} D\left(\theta_{s} \omega\right) I\left(\theta_{s} \omega\right) e^{-\int_{s}^{0} D\left(\theta_{\tau} \omega\right) d \tau} d s,
$$

which converges to $x^{*}(\omega)$ as $t \rightarrow \infty$. According to the definition of $\gamma(t)=y_{1}(t) / y(t), y(t)$ cannot take value 0 . However, $x(t)=x^{*}(w), y(t)=0$ corresponds to $x(t)=x^{*}(w), y_{1}(t)=y_{2}(t)=0$ in original system (4)-(6), representing the extinction of microorganisms.

(ii) Again let $z(t):=c x(t)+a y(t)$. On the one hand by (31),

$$
\frac{d z(t)}{d t} \leq c d i(1+\varepsilon)^{2}-d(1-\varepsilon) \alpha^{*} z(t)
$$


On the other hand since $\gamma(t)<1$ and $\nu-c b \nu \gamma(t) / a>0$, we derive from (30) that

$$
\begin{aligned}
\frac{d z(t)}{d t} & \geq c D\left(\theta_{t} \omega\right) /\left(\theta_{t} \omega\right)-D\left(\theta_{t} \omega\right) \cdot c x(t)-\left(\nu+D\left(\theta_{t} \omega\right)-\frac{c b \nu}{a} \gamma(t)\right) \cdot a y(t) \\
& \geq c D\left(\theta_{t} \omega\right) /\left(\theta_{t} \omega\right)-\left(\nu+D\left(\theta_{t} \omega\right)-\frac{c b \nu}{a} \gamma(t)\right) \cdot z(t) \\
& \geq c d i(1-\varepsilon)^{2}-\left(\nu+d(1+\varepsilon)-\frac{c b \nu}{a} \beta^{*}\right) z(t) .
\end{aligned}
$$

Denote by

$$
I_{z}:=\frac{a c d i(1-\varepsilon)^{2}}{a \nu+a d(1+\varepsilon)-c b \nu \beta^{*}} ; \quad u_{z}:=\frac{c i(1+\varepsilon)^{2}}{(1-\varepsilon) \alpha^{*}},
$$

we have the upper and lower bounds for $z(t)$ as

$$
I_{z} \leq z(t) \leq u_{z}
$$

For $0<\varepsilon<1$, define $Q_{\varepsilon}$ to be the quadrilateral

$$
Q_{\varepsilon}:=\left\{(x, y) \in \mathbb{R}_{+}^{2}: x \geq 0, y \geq 0, I_{z} \leq c x+a y \leq u_{z}\right\} .
$$

On each of the four edges of $Q_{\varepsilon}$ we have:

$$
\begin{aligned}
& \left.\frac{d x(t)}{d t}\right|_{x=0}=D\left(\theta_{t} \omega\right) I\left(\theta_{t} \omega\right)+b \nu \gamma(t) y(t)>0 \\
& \left.\frac{d z(t)}{d t}\right|_{z=I_{z}}>c d i(1-\varepsilon)^{2}-\left(\nu+d(1+\varepsilon)-\frac{c b \nu}{a} \beta^{*}\right) I_{z}=0 \\
& \left.\frac{d z(t)}{d t}\right|_{z=u_{z}}<c d i(1+\varepsilon)^{2}-d(1-\varepsilon) \alpha^{*} I_{u}=0 \\
& \left.\frac{d y(t)}{d t}\right|_{y=0}=0
\end{aligned}
$$

It shows that $y=0$ is invariant. But for $y=\epsilon \ll 1, x(t)$ satisfies $I_{z} / c \leq x(t) \leq u_{z} / c$. When condition (29) is satisfied, since function $\frac{x}{m+x}$ is increasing with respect to $x$, we have

$$
\begin{aligned}
\left.\frac{d y(t)}{d t}\right|_{y=\epsilon} & =\left(-\nu-D\left(\theta_{t} \omega\right) \gamma(t)+\frac{c x}{m+x}\right) \epsilon \\
& \geq\left(-\nu-d(1+\varepsilon) \beta^{*}+\frac{c l_{z}}{m+u_{z}}\right) \epsilon>0 .
\end{aligned}
$$

This implies that the pullback attractor $\mathcal{A}$ also contains points strictly inside the positive quadrant in addition to the set $\left(x^{*}(\omega), 0\right)$.

\section{Closing remarks}

During the past two decades, the theory of random dynamical system (RDS) has made substantial progress in describing the asymptotic behavior of systems with stochasticity, e.g. [25, 11, 9, 3, 5, 8] and references therein. The basic concept for RDS theory is to view an RDS as consisting of two ingredient - a stochastic but autonomous "noise process", and a classical dynamical system driven by this process. The RDS theory thus provides an integration of classical ergodic theory with modern dynamical systems, giving a theoretical framework parallel to classical smooth and topological dynamics (stability, attractors, bifurcation theory, etc.), while allowing one to treat in a unified way the most important classes of dynamical systems with randomness random (or stochastic) differential (or partial differential) equations [20].

Our motivation for studying chemostat models with random inputs arises from the need to provide foundations for a constructive theory of competition models exposed to external random forces. The resulting system is a system of coupled random ordinary differential equations with randomly varying input parameters, and generates a random dynamical system. Comparing to existing literatures on chemostat models, our major contributions include (1) varying randomly both the supply rate and the input concentration of the microorganism, and (2) taking into account the tendency of microorganisms to attach onto the wall.

In summary, we proved the existence of a unique random attractor to the random chemostat models. In addition, we provided geological/biological/statistical insight of the random attractor by constructing sufficient conditions for extinction and persistence 
of the microorganism. More precisely, we provided conditions on the parameters under which the random attractor contains only one "stochastic equilibrium", or contains other points strictly inside the positive quadrant besides the "stochastic equilibrium". The stochastic equilibrium here refers to a singleton set which is essentially a stochastic process, and can be regarded as the analogue of a steady state solution in deterministic systems.

Finally, we would like to mention that our objective has been to illustrate our results here in relatively basic chemostat models to place emphasize on the new features that arise due to the noise. These will also be present in more complicated and maybe more realistic models, but perhaps not so transparent.

\section{Acknowledgments}

This work has been partially supported by the Spanish Ministerio de Economía y Competitividad project MTM2011-22411, and the Consejería de Innovación, Ciencia y Empresa (Junta de Andalucía) under grant 2010/FQM314 and Proyecto de Excelencia P12-FQM-1492.

\section{References}

1. G. D'ans, P. V. Kokotovic and D. Gottlieb, A nonlinear regulator problem for a model of biological waste treatment, IEEE Transactions on Automatic Control AC-16 (1971), 341-347.

2. L. Arnold, Random Dynamical Systems, Springer-Verlag, Berlin, 1998.

3. L. Arnold and B. Schmalfuss, Fixed points and attractors for random dynamical systems. In Advances in nonlinear stochastic mechanics (Trondheim, 1995), 19-28, Solid Mech. Appl. 47 Kluwer Acad Publ., Dordrecht, 1996.

4. Y. Asai and P.E. Kloeden, Numerical schemes for random ODEs via stochastic differential equations, Commun. Appl. Analysis 17 (2013), no. 3 \& 4, 521-528.

5. P. W. Bates, H. Lisei and K. Lu, Attractors for stochastic lattice dynamical systems, Stochastics and Dynamics 6 (2006), 1-21.

6. H. R. Bungay and M. L. Bungay, Microbial interactions in continuous culture, Advances in Applied Microbiology 10 (1968), 269-290.

7. T. Caraballo, P.E. Kloeden and B. SchmalfuB, Exponentially stable stationary solutions for stochastic evolution equations and their perturbation. Appl. Math. Optim., 50 (2004), 183-207.

8. T. Caraballo, G. Lukaszewicz and J. Real, Pullback attractors for asymptotically compact nonautonomous dynamical systems, Nonlinear Analysis TMA 6 (2006), 484-498.

9. H. Crauel, A. Debussche and F. Flandoli, Random attractor J. Dynam. Differential Equations 9 (1997), 307-341.

10. A. Cunningham and R. M. Nisbet, Transients and oscillations in continuous cultures, Mathematics in Microbiology, 77-103, Academic Press, London, 1983.

11. A. Debussche, On the finite dimensionality of random attractors, Stochastic Anal. Appl. 15 (1997), 473-491.

12. F. Flandoli and B. Schmalfuss, Random attractors for the 3D stochastic Navier-Stokes equation with multiplicative noise, Stochastics Stochastics Rep. 59 (1996), no. 1-2, 21-45

13. A. G. Fredrickson and G. Stephanopoulos, Microbial competition, Science 213 (1981), no. 4511, 972-979.

14. R. Freter, Mechanisms that control the microflora in the large intestine, in Human Intestinal Microflora in Health and Disease, 33-54, D. J. Hentges, ed., Academic Press, New York, 1983.

15. R. Freter, An understanding of colonization of the large intestine requires mathematical analysis, Microecology and Therapy 16 (1986), $147-155$.

16. H. W. Jannash and R. T. Mateles, Experimental bacterial ecology studies in continuous culture, Advances in Microbial Physiology 11 (1974), 165-212.

17. P. E. Kloeden and T. Lorenz, Mean-square random dynamical systems, J. Differential Equations 253 (2012), $1422-1438$.

18. P.E. Kloeden and T. Lorenz, Pullback incremental attraction, Nonautonomous \& Random Dynamical Systems (2013), 53-60 DOI: 10.2478 /msds-2013-0004

19. P. E. Kloeden and M. Rasmussen, Nonautonomous dynamical systems, American Mathematical Society Providence, RI (2011)

20. M. Marcondes de Freitas and E. D. Sontag, Random dynamical system with inputs. In Nonautonomous dynamical systems in the life sciences, P. E. Kloeden and C. Pötzsche (eds.), Lecture Notes in Mathematics, 2102 (2013), 41-87.

21. J. W. M. La Riviere, Microbial ecology of liquid waste, Advances in Microbial Ecology 1 (1977), 215-259.

22. V. Sree Hari Rao and P. Raja Sekhara Rao, Dynamic Models and Control of Biological Systems, Springer-Verlag, Heidelberg, 2009.

23. H. L. Smith, Monotone Dynamical Systems: an Introduction to the Theory of Competitive and Cooperative Systems, Mathematical Surveys and Monogrpahs, 41. American Mathematical Society, Providence, RI, 1995.

24. H. L. Smith and P. Waltman, The Theory of the Chemostat: Dynamics of Microbial Competition, Cambridge University Press , Cambridge, UK (1995).

25. B. Schmalfuss, The random attractor of the stochastic Lorenz system, Z. Angew. math. Phys. 48 (1997), 951-975.

26. P. A. Taylor and J. L. Williams, Theoretical studies on the coexistence of competing species under continuous flow conditions, Canadian Journal of Microbiology 21 (1975), 90-98.

27. H. Veldcamp, Ecological studies with the chemostat, Advances in Microbial Ecology 1 (1977), 59-95.

28. P. Waltman, Competition Models in Population Biology, CBMS-NSF Regional Conference Series in Applied Mathematics, 45. Society for Industrial and Applied Mathematics, Philadelphia, 1983.

29. P. Waltman, Coexistence in chemostat-like model, Rocky Mountain Journal of Mathematics 20 (1990), 777-807.

30. P. Waltman, S. P. Hubbel and S. B. Hsu, Theoretical and experimental investigations of microbial competition in continuous culture, Modeling and Differential Equations in Biology (Conf., Southern Illinois Univ. Carbondale, III., 1978), pp. 107-152. Lecture Nots in 
Pure and Appl. Math., 58, Dekker, New York, 1980.

31. F. M. Williams Dynamics of microbial populations. In Systems Analysis and Simulation in Ecology Vol. 1, pp. 197-265, B.O. Patten (Ed.), Academic Press, New York, 1971. 